\title{
Computing nucleon EDM on a lattice
}

\author{
Michael Abramczyk ${ }^{1}$, Sinya Aoki ${ }^{2,3}$, Tom Blum ${ }^{1,4}$, Taku Izubuchi ${ }^{4,5}$, Hiroshi Ohki ${ }^{4}$, and Sergey \\ Syritsyn $n^{4,6, \star}$ \\ ${ }^{1}$ Physics Department, University of Connecticut, Storrs, Connecticut 06269, USA \\ ${ }^{2}$ Center for Gravitational Physics, Yukawa Institute for Theoretical Physics, Kyoto University, Kyoto 606-8502, \\ Japan \\ ${ }^{3}$ Center for Computational Sciences, University of Tsukuba, Tsukuba 305-8577, Japan \\ ${ }^{4}$ RIKEN/BNL Research Center, Brookhaven National Laboratory, Upton, New York 11973, USA \\ ${ }^{5}$ Physics Department, Brookhaven National Laboratory, Upton, New York 11973, USA \\ ${ }^{6}$ Department of Physics and Astronomy, Stony Brook University, Stony Brook, New York 11794, USA
}

\begin{abstract}
I will discuss briefly recent changes in the methodology of computing the baryon EDM on a lattice. The associated correction substantially reduces presently existing lattice values for the proton and neutron theta-induced EDMs, so that even the most precise previous lattice results become consistent with zero. On one hand, this change removes previous disagreements between these lattice results and the phenomenological estimates of the nucleon EDM. On the other hand, the nucleon EDM becomes much harder to compute on a lattice. In addition, I will review the progress in computing quark chromo-EDM-induced nucleon EDM using chiral quark action.
\end{abstract}

\section{Introduction}

Observing a non-zero nucleon electric dipole moment (EDM) will be evidence of violations of fundamental P,T-symmetries beyond the level of the Standard Model. Such violations are necessary to explain the excess of matter over antimatter in the Universe, and the CP in the CKM matrix of the Standard model is insufficient for the observable amount of matter. Knowledge of nucleon structure and interactions is necessary to interpret constraints from precise experiments attempting to measure the EDM of nucleons and nuclei, the precision of which is projected to improve by two orders of magnitude in the next decade.

An EDM may be induced within the Standard Model by the QCD $\bar{\theta}$-term, the smallness of which is known as the strong $C P$ problem. In order to constrain $\bar{\theta}_{\mathrm{QCD}}$ from nucleon EDM measurements, lattice calculations of the $\bar{\theta}_{\mathrm{QCD}}$-induced neutron EDMs have been performed earlier in Refs. [1-3] using the background electric field and in Refs. [4-10] from nucleon matrix elements in $C P$ vacuum. In our recent paper [11], we have found that the analysis of the nucleon EDM in Refs. [4-10] was flawed, and all previous results are largely consistent with zero.

\footnotetext{
${ }^{\star}$ Speaker, e-mail: sergey.syritsyn@ @stonybrook.edu
} 
In this report, we calculate the nucleon EDM induced by the $C P$-odd quark-gluon electric dipole interaction (so-called chromo-EDM)

$$
\mathcal{L}_{c E D M}=i \sum_{\psi=u, d} \frac{\tilde{\delta}_{\psi}}{2} \bar{\psi} T^{a}\left(g_{S} G_{\mu \nu}^{a}\right) \sigma^{\mu \nu} \gamma_{5} \psi=i \sum_{\psi=u, d} \tilde{\delta}_{\psi} O_{\psi \tilde{G}}, \quad O_{\psi \tilde{G}}=\frac{1}{2} \bar{\psi} T^{a}\left(g_{S} G_{\mu \nu}^{a}\right) \sigma^{\mu \nu} \gamma_{5} \psi,
$$

using $24^{3} \times 64$ lattices generated with Iwasaki gauge action and $N_{f}=2+1$ dynamical flavors of Domain Wall fermions by the RBC collaboration. These lattices have pion mass $m_{\pi} \approx 330 \mathrm{MeV}$ and lattice spacing $a^{-1} \approx 1.73 \mathrm{GeV}$. We perform these calculations using two independent methods: (1) nucleon matrix elements of the vector current and (2) nucleon energy shifts in the background electric field.

\section{Nucleon EDM on a lattice}

In order to compute the nucleon EDM on a lattice, one has to calculate nucleon matrix elements of the quark vector current in a $C P$ vacuum $\left\langle N\left|J^{\mu}\right| N\right\rangle_{C P}$. $C P$ violation leads to "parity mixing" of the nucleon spinor $u \rightarrow \tilde{u}=e^{i \alpha_{5} \gamma_{5}} u$, which is equivalent to a chiral rotation of the nucleon mass $m_{N} \rightarrow e^{-2 i \alpha_{5} \gamma_{5}} m_{N}$ in the Dirac equation. In order to extract the nucleon matrix elements, one has to analyze the nucleon-current correlators as

$$
\left\langle N\left(p^{\prime}\right) J^{\mu} \bar{N}(p)\right\rangle \propto e^{i \alpha_{5} \gamma_{5}} \frac{-i \not p_{\mathcal{E} u c}^{\prime}+m_{N}}{2 E_{N}^{\prime}}\left\{e^{i \alpha_{5} \gamma_{5}}\right\}^{?} \Gamma_{\mathcal{E} u c}^{\mu}\left\{e^{i \alpha_{5} \gamma_{5}}\right\}^{?} \frac{-i \not p_{\mathcal{E} u c}+m_{N}}{2 E_{N}} e^{i \alpha_{5} \gamma_{5}},
$$

where the phase factors in curly braces were deemed necessary as parts of the nucleon propagator in the CP vacuum in Refs. [4-10]. However, as we discuss in our recent paper [11], these factors lead to incorrect results and should be omitted because the correct decomposition of the vector current into $C P$-even and -odd form factors is

$$
\left\langle p+q, \sigma^{\prime}\left|J^{\mu}\right| p, \sigma\right\rangle=\bar{u}_{p+q, \sigma^{\prime}}\left[F_{1} \gamma^{\mu}+\left(F_{2}+i F_{3} \gamma_{5}\right) \frac{i \sigma^{\mu v} q_{v}}{2 m_{N}}\right] u_{p, \sigma}
$$

with spinors satisfying the Dirac equation $\left(i \not \supset-m_{N}\right) u$ and having definite parity $\gamma_{4} u_{\vec{p}}=+u_{(-\vec{p})}$. The improper chiral phase factors $\left\{e^{i \alpha_{5} \gamma_{5}}\right\}$ in Eq. 2 lead to mixing of form factors $F_{3}$ and $F_{2}$, resulting in a spurious contribution to the electric dipole form factor

$$
F_{3} \rightarrow \tilde{F}_{3}=F_{3}-2 \alpha_{5} F_{2} \text {. }
$$

(conventions are consistent with our definitions in Ref. [11]).

\section{EDM from form factors}

In this section we summarize briefly our methodology, with full details provided in Ref. [11]. The $C P$-odd $(\overline{C P})$ form factor $F_{3}$ is extracted from nucleon matrix elements in presence of $\overline{C P}$ interactions with the action $S_{C P-\text { even }}+i \delta^{\overline{C P}} S=S_{C P-\text { even }}+i \tilde{\delta}_{\psi} \sum_{x}\left[O_{\psi \tilde{G}}\right]_{x}$. To compute them, we evaluate corrections to the nucleon correlation functions $C^{C P}=C^{C P \text {-even }}-i \tilde{\delta}_{\psi} \delta^{\overline{C P}} C$ linear in the $C P$-odd operator $O_{\psi \tilde{G}}$ :

$$
\begin{aligned}
\delta^{\overline{C P}} C_{N J \bar{N}}\left(\vec{p}^{\prime}, \vec{q} ; t_{\mathrm{sep}}, t_{\mathrm{op}}\right) & =\sum_{x, \vec{y}, \vec{z}} e^{-i \vec{p}^{\prime} \vec{y}+i \vec{q} \vec{z}}\left\langle N\left(\vec{y}, t_{\mathrm{sep}}\right)\left[\bar{q} \gamma^{\mu} q\right]_{\left(\vec{z}, t_{o p}\right)} \bar{N}(0)\left[O_{\psi \tilde{G}}\right]_{x}\right\rangle \\
\delta^{\overline{C P}} C_{N \bar{N}}\left(\vec{p} ; t_{\mathrm{sep}}\right) & =\sum_{x, \vec{y}} e^{-i \vec{p}^{\prime} \vec{y}}\left\langle N\left(\vec{y}, t_{\mathrm{sep}}\right) \bar{N}(0)\left[O_{\psi \tilde{G}}\right]_{x}\right\rangle
\end{aligned}
$$



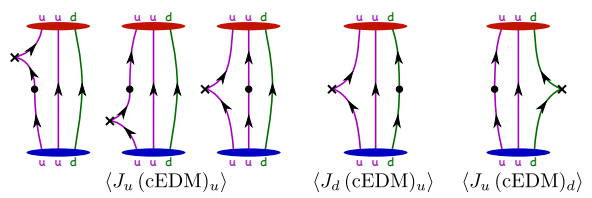

(a)
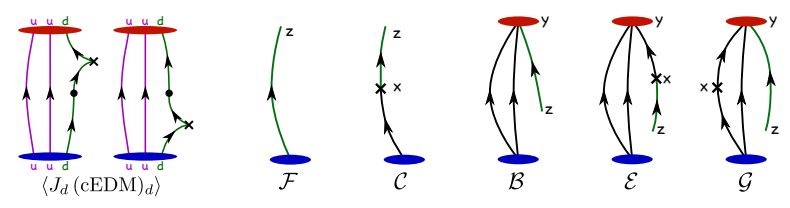

(b)

Figure 1. (a) Quark-connected contractions of the nucleon, quark current (dots), and cEDM operators (crosses). (b) Propagators required for computing quark-connected contractions of the nucleon, quark current, and cEDM operators.

in addition to the regular $C P$-even correlators $C_{N \bar{N}}=\langle N \bar{N}\rangle$ and $C_{N J \bar{N}}=\left\langle N\left[\bar{q} \gamma^{\mu} q\right] \bar{N}\right\rangle$. Connected insertions of $O_{\psi \tilde{G}}$ generate contractions (shown in Fig. 1(a) for nucleon-current correlators) that are computed using a set of sequential propagators (Fig. 1(b)). The gluon field strength $T^{a}\left[g_{S} G_{\mu \nu}^{a}\right]_{x}^{\text {cont }}$ in the lattice chromo-EDM operator is approximated using a "clover" plaquettes centered at site $x$,

$$
\left(T^{a}\left[g_{S} G_{\mu \nu}^{a}\right]_{x}^{\text {cont }}\right) \rightarrow\left[G_{\mu \nu}\right]^{\text {clov }}=\frac{1}{8 i}\left[\left(U_{x,+\hat{\mu},+\hat{v}}^{P}+U_{x,+\hat{v},-\hat{\mu}}^{P}+U_{x,-\hat{\mu},-\hat{v}}^{P}+U_{x,-\hat{v}, \hat{\mu}}^{P}\right)-\text { h.c. }\right]
$$

Nucleon energies in $C P$-even vacuum are extracted from the $\langle N \bar{N}\rangle$ correlator with 2 -state fits (Fig. 2(a)). As widely discussed in the literature, in a $C P$ vacuum the lattice nucleon operator $\left(u^{T} C \gamma_{5} d\right) u$ creates a parity-mixed state so that the nucleon propagator is

$$
\left.C_{N \bar{N}}^{C P}(\vec{p}, t)\right|_{g . s .}=\frac{-i \not p_{\varepsilon u c}+m_{N} e^{2 i \alpha_{5} \gamma_{5}}}{2 E_{N}} e^{-E_{N} t}=e^{i \alpha_{5} \gamma_{5}} \frac{-i \not p_{\mathcal{E} u c}+m_{N}}{2 E_{N}} e^{i \alpha_{5} \gamma_{5}} e^{-E_{N} t} .
$$

where $\left(-i \not p_{\mathcal{E} u c}\right)=E_{N} \gamma_{\mathcal{E} u c}^{4}-i \vec{p} \cdot \vec{\gamma}_{\mathcal{E} u c}$. In order to subtract the $F_{1,2}$ terms mixing with $F_{3}$, we calculate the parity-mixing angle $\alpha_{5}$ from the ratio of the nucleon correlators,

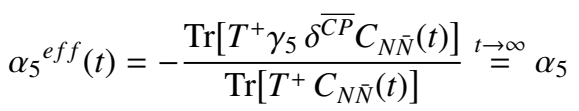

where $T^{+}=\frac{1+\gamma_{4}}{2}$, and extract the value from its plateaus (see Fig. 2(b)).

To evaluate the ground state matrix elements, we use the three central plateau points of the ratios,

$$
\left[\delta^{\overline{C P}}\right] \mathcal{R}_{N J \bar{N}}\left(t_{\text {sep }}, t_{o p}\right)=\frac{\left[\delta^{\overline{C P}}\right] C_{N J \bar{N}}\left(t_{\text {sep }}, t_{o p}\right)}{c_{2}^{\prime}\left(t_{\text {sep }}\right)} \sqrt{\frac{c_{2}^{\prime}\left(t_{\text {sep }}\right)}{c_{2}\left(t_{\text {sep }}\right)} \frac{c_{2}^{\prime}\left(t_{o p}\right)}{c_{2}\left(t_{o p}\right)} \frac{c_{2}\left(t_{\text {sep }}-t_{o p}\right)}{c_{2}^{\prime}\left(t_{\text {sep }}-t_{o p}\right)}},
$$

where $c_{2}^{(\prime)}(t)=\operatorname{Tr}\left[T^{+} C_{N \bar{N}}\left(\vec{p}^{(\prime)}, t\right)\right]$ with a fixed sink momentum $\vec{p}^{\prime}=\overrightarrow{0}$ and varying source momenta $\vec{p}$. Form factors $F_{1,2}$ are extracted with overdetermined analysis of matrix elements of the quark vector current averaged over directions of the current and the momentum transfer $\vec{q}=\left(\vec{p}^{\prime}-p\right)$ that give equivalent combinations of $F_{1,2}$ as described, e.g., in Ref. [12]. The results for the Sachs form factors $G_{E}=F_{1}-\tau F_{2}$ and $G_{M}=F_{1}+F_{2}$ (where $\tau=\frac{Q^{2}}{4 m_{N}^{2}}=\frac{E_{N}-m_{N}}{2 m_{N}}$ ) are shown in Fig. 3(a) and demonstrate that there is no observable $t_{\text {sep }}$ dependence indicating no substantial contamination from excited states. The electric dipole form factor $F_{3}$ can be extracted from both timelike and spacelike current. We find that the time component $\left\langle N J^{4} \bar{N}\right\rangle$ yields much better precision than the spacelike component $\left\langle N J^{3} \bar{N}\right\rangle$. 


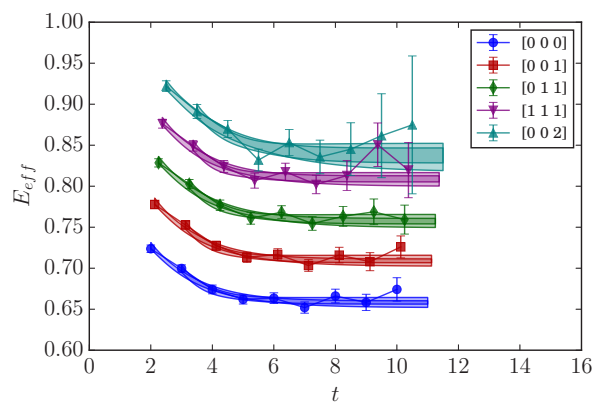

(a)

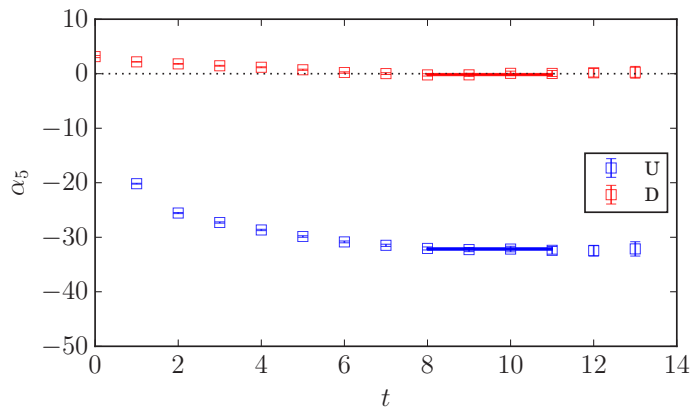

(b)

Figure 2. (a) Effective energy plots for $\vec{p}^{2} \leq 4 p_{\text {min }}^{2}$, together with two-state fits. (b) Chiral rotation angle $\alpha_{5}$ of the proton field induced by $u$ - and $d$-quark cEDM interactions (no disconnected contractions, no renormalization);

Combining these components did not result in improved precision of the $F_{3}$ form factor. With only the $\left\langle N J^{4} \bar{N}\right\rangle$ component, the form factor is given by the expression

$$
(1+\tau) F_{3}\left(Q^{2}\right)=\frac{m_{N}}{q_{3} \mathcal{K}_{\mathcal{R}}} \operatorname{Tr}\left[T_{S_{z}+}^{+} \delta^{\overline{C P}} \mathcal{R}_{N J^{4} \bar{N}}\right]-\alpha_{5} G_{E}\left(Q^{2}\right)
$$

where $T_{S_{z}+}^{+}=T^{+}\left(1+\Sigma_{z}\right)=\frac{1}{2}\left(1+\gamma_{4}\right)\left(1-i \gamma_{1} \gamma_{2}\right), \tau=\frac{Q^{2}}{4 m_{N}^{2}}=\frac{E_{N}-m_{N}}{2 m_{N}}$ and $\mathcal{K}_{\mathcal{R}}=\frac{m_{N}}{\left.\sqrt{2 E_{N}\left(m_{N}+E_{N}\right.}\right)}$. The second term in the rhs is the mixing subtraction. Following our recent paper [11], we stress that the correct subtraction of $F_{1,2}$ mixing contributions from $F_{3}$ is different from the expressions used in earlier Refs. [4-10] by a term $\left(-2 \alpha_{5} F_{3}\right)$ due to spurious mixing of $F_{2}$ and $F_{3}$ in the vector current vertex in these papers. The $F_{3}$ results for the four combinations of flavors in the cEDM and current operators are shown in Fig. 3(b), again demonstrating no visible $t_{\text {sep }}$ dependence.

\section{EDM from energy shift}

Calculation of the dipole moment using a uniform background field is advantageous because it does not require momentum extrapolation. The calculation is straightforward for the neutron, but for the proton one has to analyze correlation functions that are complicated due to its constant acceleration [13]. However, since the uniform background field is quantized on a lattice, it cannot be made arbitrarily small. For small lattices, the minimal field strength is very large with the magnitude comparable to the QCD scale: $\mathcal{E}_{0}=\frac{3 \cdot 2 \pi}{a^{2} L_{x} L_{t}}$, where the extra factor of 3 is due to the fractional quark charge. On the $24^{3} \times 64$ lattice, the minimal electric field is $\mathcal{E}_{0} \approx 0.04 \mathrm{GeV}^{2} \approx 200 \mathrm{MV} / \mathrm{fm}$. Such an electric field pulls the $u$ quark in the neutron with tension $\approx(160 \mathrm{MeV})^{2}$, or approximately $14 \%$ of the QCD string tension. Although it may deform the neutron away from the ground state, we believe that the correction is insignificant at our level of precision.

We introduce the uniform electric field along the $z$ axis of the lattice as described in Ref. [11]. Using the modified QCD $+U(1)$ gauge links, we calculate the regular nucleon correlator $C_{N \bar{N}, \mathcal{E}}$ and its linear perturbation in the $C P$-odd interaction $\sum_{x}\left[O_{\psi \tilde{G}}\right]_{x}$, similarly to the form factor $F_{3}$ :

$$
\delta^{\overline{C P}} C_{N \bar{N}, \mathcal{E}}(\vec{p}, t)=\sum_{\vec{y}} e^{-i \vec{p}(\vec{y}-\vec{x})}\left\langle N(\vec{y}, t) \bar{N}(\vec{x}, 0) O_{\psi G}^{\overline{C P}}\right\rangle_{\mathcal{E}} .
$$




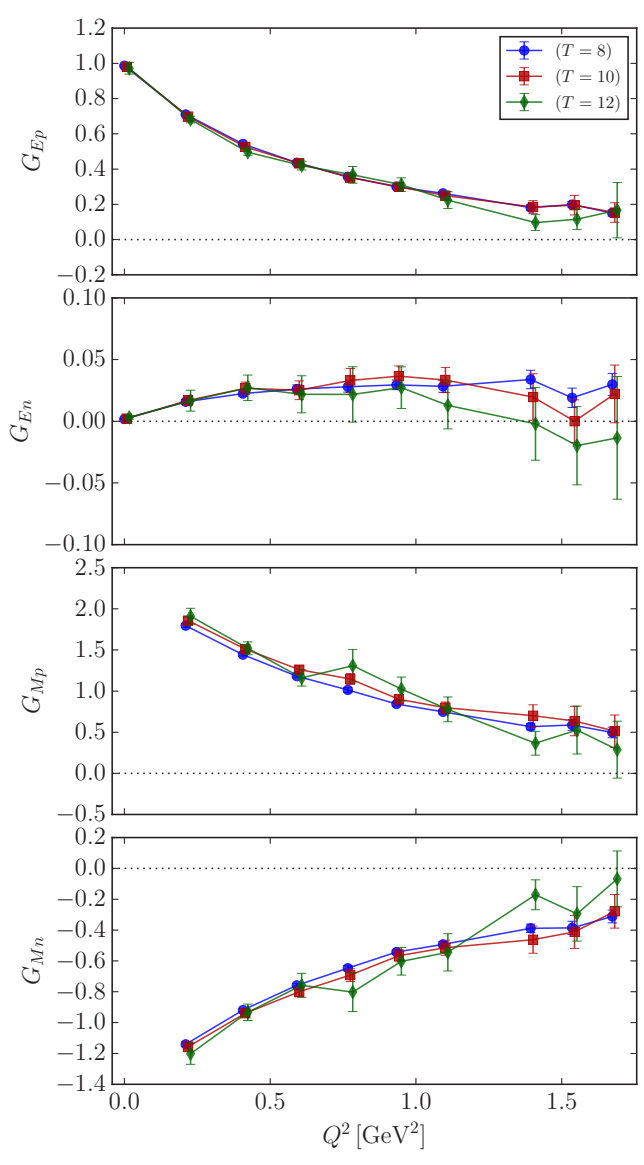

(a) P,T-even nucleon vector current (Sachs) form factors.

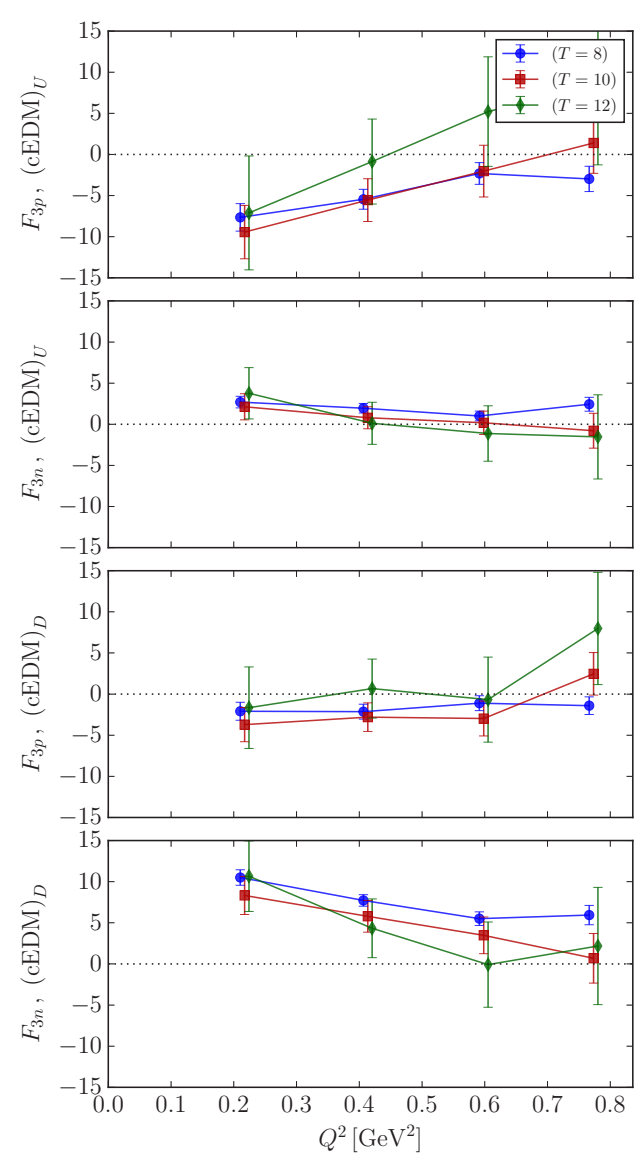

(b) Electric dipole form factors $F_{3}$ induced by $u$ - and $d$-quark chromo-EDM interactions.

Figure 3. Nucleon CP-even and CP-odd electric dipole form factors. Disconnected contractions are not included for either currents or cEDM insertions.

It is important to use the modified gauge links both in the computing of the propagators and in the construction of the smeared sources and sinks. Otherwise, since the individual quarks are charged, the results would depend on the gauge of the electromagnetic potential. For the purpose of source construction, the QCD gauge links are first APE-smeared, with the electromagnetic potential applied afterwards.

Following Ref.[11], the energy of a polarized $(\vec{S} \| \vec{E})$ particle on a Euclidean lattice is shifted by the imaginary value $\delta E=-(\zeta / 2 m) i \mathcal{E}$. The nucleon correlator at rest $(\vec{p}=0)$ is therefore

$$
C_{N \bar{N}, \mathcal{E}}^{\mathscr{C}}(\vec{p}=0, t)=\left|Z_{N}\right|^{2} e^{i \alpha_{5} \gamma_{5}} \frac{1+\gamma_{4}}{2}\left[\frac{1+\Sigma_{z}}{2} e^{-\left(m_{N}+\delta E\right) t}+\frac{1-\Sigma_{z}}{2} e^{-\left(m_{N}-\delta E\right) t}\right] e^{i \alpha_{5} \gamma_{5}} .
$$

Analogously to the treatment of the $C P$-odd form factor $F_{3}$, the linear perturbation in $\sum_{x}\left[O_{\psi \tilde{G}}\right]_{x}$ yields

$$
C_{N \bar{N}, \mathcal{E}}-i \tilde{\delta}_{\psi} \delta^{\overline{C P}} C_{N \bar{N}, \mathcal{E}} \stackrel{t \rightarrow \infty}{=}\left|Z_{N}\right|^{2} e^{-m_{N} t}\left[\frac{1+\gamma_{4}}{2}+i \alpha_{5} \gamma_{5}-\Sigma_{z} \delta E t\right]
$$




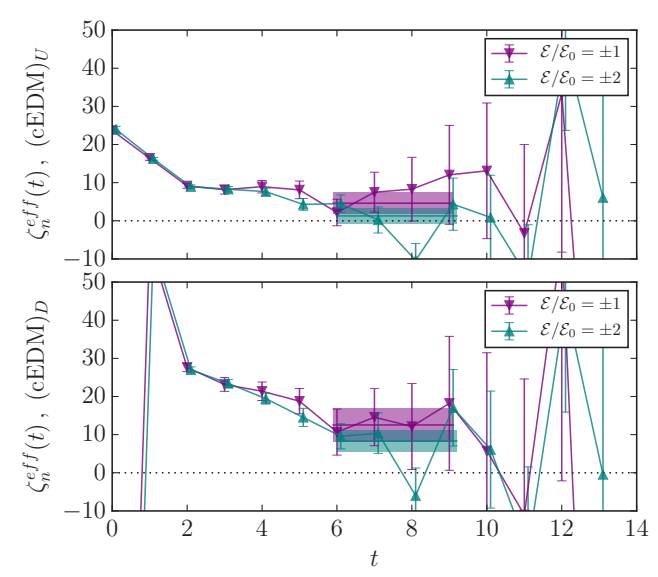

(a) The neutron EDM computed from energy shifts with two values of the electric field. The units are dimensionless and the scale is the same as for $F_{3}$.

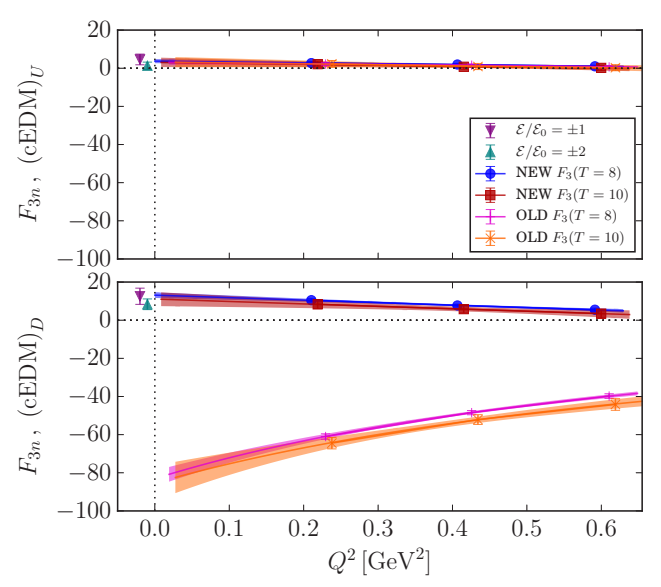

(b) Comparison of the neutron EDFF $F_{3 n}\left(Q^{2}\right)$ computed with the conventional [4-10] and the new (11) formulas to the energy shift results.

from which we obtain the following estimator for the electric dipole moment:

$$
\zeta^{\mathrm{eff}}(t)=2 m_{N} d_{N}^{\mathrm{eff}}(t)=-\frac{2 m_{N}}{\mathcal{E}_{z}}\left[R_{z}(t+1)-R_{z}(t)\right], \quad R_{z}(t)=\frac{\operatorname{Tr}\left[T^{+} \Sigma_{z} \delta^{\overline{C P}} C_{N \bar{N}, \mathcal{E}_{z}}(t)\right]}{\operatorname{Tr}\left[T^{+} C_{N \bar{N}, \mathcal{E}_{z}}(t)\right]}
$$

To estimate the effects of the ground state distortion by the background field, we have computed the neutron correlation functions with two values $\mathcal{E}=\mathcal{E}_{0}$ and $2 \mathcal{E}_{0}$, with the results for $\zeta^{\text {eff }}(t)$ shown in Fig. 4(a). We choose $t=6 \ldots 9$ as the common plateau to estimate the value of $\zeta$ for both flavors $\psi=u, d$. In the case of $d$-quark cEDM, we observe nonzero values of the energy shift. The EDM values computed with $\mathcal{E}=\mathcal{E}_{0}$ and $2 \mathcal{E}_{0}$ agree well with each other, indicating that the energy shift is linear in $\mathcal{E}$ and our current EDM results are not sensitive to the nucleon ground state distortion by the electric field.

Our calculation of the neutron EDM from the energy shift does not depend on the parity mixing (chiral phase rotation) of the nucleon states in the $C P$ vacuum. This mixing, if not taken into account correctly, may lead to the spurious mixing between $F_{3}$ and $F_{2}$. By comparing the energy shift and the form factor results, we demonstrate numerically that indeed our analysis of the parity mixing in Ref. [11] is correct. This comparison is shown in Fig. 4(b), together with form factor values $\tilde{F}_{3}$ computed using expressions from Refs. [4-10] given in Eq. 4 . The $\tilde{F}_{3}$ values are different from $F_{3}$ only if the mixing angle $\alpha_{5}$ is relatively large. Thus, in the case of $u$-quark cEDM, the value $\alpha_{5}$ for the neutron is small, and there is no observable difference between $F_{3}$ and $\tilde{F}_{3}$. However, in the case of $d$-quark cEDM, the difference is remarkable. It is clear that even with the current uncertainties due to excited states and the $Q^{2} \rightarrow 0$ extrapolation, the comparison favors agreement with $F_{3}$ but not $\tilde{F}_{3}$. This agreement between the energy shift and the form factor calculation of nEDM serves as a numerical cross-check of our analysis of the latter. We believe that this discrepancy has not been detected in earlier lattice studies of the $\bar{\theta}_{Q C D}$-induced nucleon EDM because of the small corresponding values of the mixing angle as well as the relatively large statistical errors, which made the comparison between Refs. [2] and [4] inconclusive. 


\section{Summary}

We have performed calculations of the nucleon electric dipole moments induced by $C P$-odd quarkgluon electric dipole interactions using two different methods. In the first method we calculate nucleon matrix elements in the $C P$ vacuum by evaluating up to four-point connected nucleon correlation functions. The $C P$-odd interaction is treated as perturbation of the $C P$-even vacuum and is straightforward to analyze using sequential propagators. We have performed calculations with three different source-sink separations and we do not observe any significant excited states in either the $C P$-even form factors $F_{1,2}$ or the $C P$-odd electric dipole form factor $F_{3}$.

In the second method, we also treat the $C P$-odd interaction as a perturbation but compute the neutron EDM from the energy shift in a uniform background electric field. The energy-shift method for computing the nucleon EDM has been used before [1-3], but our calculation is the first one that uses the uniform background electric field respecting periodic boundary conditions [13]. The statistics is identical in both methods, and the energy shift results agree with the form factors $F_{3}$ if they are computed with the new formula [11]. Also, both methods yield comparable uncertainty. The energyshift method may be preferable in the future because it does not require forward-limit extrapolation, and excited states may be easier to control [14].

In both methods, we observe statistically significant signal for the cEDM-induced nucleon EDM, although we do not include disconnected diagrams in our calculation, and our results are not yet renormalized. Comparison of the results from the two methods serve as a numerical cross-check of our recent finding that the extraction of the $C P$-odd form factor $F_{3}$ and the nucleon EDM in previous works was incorrect. Specifically, the reported values contain spurious contributions $\left(-2 \alpha_{5} \kappa\right)$ due to mixing with the anomalous magnetic moment $\kappa$ of the nucleon.

We plan to calculate disconnected contributions to the $F_{3}$ form factors as an extension to the presented work, combining the existing nucleon correlators and quark-disconnected loops with insertions of the quark current, chromo-EDM, and both. Although the disconnected contractions are simpler to construct (they do not require four-point correlators and associated sequential propagators), we expect that the stochastic noise will be a much bigger problem compared to the connected contractions.

\section{ACKNOWLEDGEMENTS}

T. B. is supported by U.S. Department of Energy Grant No. DE-FG02-92ER40716. T. I. is supported in part by U.S. Department of Energy Contracts No. AC-02-98CH10886 and No. DE-SC0012704. S. A. and T. I. are also supported in part by the Grant-in-Aid of the Japanese Ministry of Education, Sciences and Technology, Sports and Culture (MEXT) for Scientific Research (No. JP16H03978, No. JP17H02906, and No. 26400261), by a priority issue (Elucidation of the Fundamental Laws and Evolution of the Universe) to be tackled by the "Post-K" Computer, and by the Joint Institute for Computational Fundamental Science (JICFuS). H. O. is supported by the RIKEN Special Postdoctoral Researcher program. S. S. is supported by the Nathan Isgur fellowship program at JLab and by RIKEN BNL Research Center under its joint tenure track fellowship with Stony Brook University. S. A. and S. S. are also grateful for the hospitality of the Kavli Institute for Theoretical Physics (UC Santa Barbara) during the "Nuclear16" workshop. This research was supported in part by the National Science Foundation under Grant No. NSF PHY11-25915. Gauge configurations with dynamical domain wall fermions used in this work were generated by the RBC/UKQCD Collaboration. The computation was performed using the Hokusai supercomputer of the RIKEN ACCC facility and the Jlab cluster as part of the USQCD Collaboration. This material is based upon work supported by the U.S. Department of Energy, Office of Science, Office of Nuclear Physics under Contract No. DEAC05-06OR23177. 


\section{References}

[1] S. Aoki, A. Gocksch, Phys. Rev. Lett. 63, 1125 (1989), [Erratum: Phys. Rev. Lett.65,1172(1990)]

[2] E. Shintani, S. Aoki, N. Ishizuka, K. Kanaya, Y. Kikukawa et al., Phys.Rev. D75, 034507 (2007), hep-lat/0611032

[3] E. Shintani, S. Aoki, Y. Kuramashi, Phys.Rev. D78, 014503 (2008), 0803.0797

[4] E. Shintani, S. Aoki, N. Ishizuka, K. Kanaya, Y. Kikukawa et al., Phys.Rev. D72, 014504 (2005), hep-lat/0505022

[5] F. Berruto, T. Blum, K. Orginos, A. Soni, Phys.Rev. D73, 054509 (2006), hep-1at/0512004

[6] R. Horsley, Y. Nakamura, D. Pleiter et al., The Electric dipole moment of the nucleon from simulations at imaginary vacuum angle theta (2008), 0808.1428

[7] F.K. Guo, R. Horsley, U.G. Meissner, Y. Nakamura, H. Perlt, P.E.L. Rakow, G. Schierholz, A. Schiller, J.M. Zanotti, Phys. Rev. Lett. 115, 062001 (2015), 1502.02295

[8] A. Shindler, T. Luu, J. de Vries, Phys. Rev. D92, 094518 (2015), 1507.02343

[9] C. Alexandrou, A. Athenodorou, M. Constantinou, K. Hadjiyiannakou, K. Jansen, G. Koutsou, K. Ottnad, M. Petschlies, Phys. Rev. D93, 074503 (2016), 1510.05823

[10] E. Shintani, T. Blum, T. Izubuchi, A. Soni, Phys. Rev. D93, 094503 (2016), 1512 . 00566

[11] M. Abramczyk, S. Aoki, T. Blum, T. Izubuchi, H. Ohki, S. Syritsyn, Phys. Rev. D96, 014501 (2017), 1701.07792

[12] S. Syritsyn, J. Bratt, M. Lin, H. Meyer, J. Negele et al., Phys.Rev. D81, 034507 (2010), Q907. 4194

[13] W. Detmold, B.C. Tiburzi, A. Walker-Loud, Phys. Rev. D79, 094505 (2009), 0904 . 1586

[14] C. Bouchard, C.C. Chang, T. Kurth, K. Orginos, A. Walker-Loud (2016), 1612.06963 